\title{
Analysis of the Impact of Isoquinoline Alkaloids, Derived from Macleaya cordata Extract, on the Development and Innate Immune Response in Swine and Poultry
}

\author{
Hengjia Ni, ${ }^{1,2}$ Yordan Martínez, ${ }^{1,3}$ Guiping Guan,,2 Román Rodríguez, ${ }^{3}$ \\ Dairon Más, ${ }^{3}$ Hanhui Peng, ${ }^{2}$ Manuel Valdivié Navarro, ${ }^{4}$ and Gang Liu ${ }^{1}$ \\ ${ }^{1}$ Key Laboratory of Agro-Ecological Processes in Subtropical Region, Institute of Subtropical Agriculture, \\ Chinese Academy of Sciences, Hunan Provincial Engineering Research Center of Healthy Livestock, \\ Scientific Observing and Experimental Station of Animal Nutrition and Feed Science in South-Central, \\ Ministry of Agriculture, Hunan Co-Innovation Center of Animal Production Safety, Hunan 410125, China \\ ${ }^{2}$ College of Bioscience and Biotechnology, Hunan Agricultural University, Changsha, Hunan 410128, China \\ ${ }^{3}$ Centro de Estudios de Producción Animal, Universidad de Granma, Apartado Postal 21, Bayamo, 85100 Granma, Cuba \\ ${ }^{4}$ Instituto de Ciencia Animal, Apartado Postal 24, San José de Las Lajas, Mayabeque, Cuba \\ Correspondence should be addressed to Gang Liu; gangle.liu@gmail.com
}

Received 30 June 2016; Accepted 24 October 2016

Academic Editor: Yang Zhou

Copyright (C) 2016 Hengiia Ni et al. This is an open access article distributed under the Creative Commons Attribution License, which permits unrestricted use, distribution, and reproduction in any medium, provided the original work is properly cited.

\begin{abstract}
Medicinal extract has been chronicled extensively in traditional Chinese medicine. Isoquinoline alkaloids, extract of Macleaya cordata (Willd.) R. Br., have been used as feed additive in both swine and poultry. Dietary supplementation with isoquinoline alkaloids increases feed intake and weight gain. In addition, recent researches have demonstrated that isoquinoline alkaloids can regulate metabolic processes, innate immune system, and digestive functioning in animals. This review summarizes the latest scientific researches on isoquinoline alkaloids which are extracted from Macleaya cordata (Willd.) R. Br. This review specifically focuses on its role as a feed supplement and its associated impact on growth performance and innate immune system, as well as its capacity to act as a substitute for oral antibiotics.
\end{abstract}

\section{Introduction}

Macleaya cordata (Willd.) R. Br., also known as Bocconia cordata or plume poppy, belongs to the Papaveraceae family. It is an herbaceous perennial plant, ubiquitously dispersed in central and southeastern China. It is also found in the regions where the parasitic disease (schistosomiasis) is prevalent $[1,2]$.

Macleaya cordata (Willd.) R. Br. contains a number of important alkaloids, which include sanguinarine (SG), dihydroderivative (DHSG), chelerythrine $(\mathrm{CH})$, protopine (PR), allocryptopine (AL), and phenolic acids [3, 4]. A small amount of other isoquinoline alkaloids have also been traced in this plant, such as chelirubine, macarpine, sanguidimerine, chelidimerine, homochelidonine, cryptopine, berberine, coptisine, chelilutine, bocconarborine A, bocconarborine $\mathrm{B}$, oxysanguinarine, norsanguinarine, angoline, bocconoline, 6ethoxychelerythrine, 6-ethoxysanguinarine, protopine-Noxide, 6-methoxydihydrosanguinarine, 6-acetonyl-dihyrochelerythrine, and 6-acetonyl-dihydrosanguinarine [3].

Macleaya cordata (Willd.) R. Br. grow above the ground and have been used as traditional Chinese medicine for a long time. They are utilized for specific purposes, such as pain relief, modification of the immune system, and reduction of inflammation. The capacity to suppress the proliferation of bacteria, fungi, and viruses [5] has been ascribed to the quaternary benzo[c]phenanthridine alkaloids (QBA), SG and CH $[2,6,7]$. Furthermore, its positive effects on health are evidenced by its ability to inhibit the growth of microorganisms, to block the release or action of adrenaline at nerve endings, to decrease the excitation of sympathetic nervous system, to prevent from fungal infections, and to 
be used in the treatment of cancer. It also can act as an antiseptic compound, a pesticide against molluscs, and an agent to destroy plant-parasitic nematode worms [2, 8-11].

More recently, food supplements derived from plants have been fed to farm animals. Gradually, they have evoked attention as a substitute to antibiotic growth promoters [12]. This is attributed to the fact that these plants and their extracts are natural substances. They are found to be beneficial in improving growth performance, digestive function, and the absorption of nutrients. They are also helpful in improving the ability of anti-infection and reducing the incidence of diarrhea [12-18].

Based on these properties, Macleaya cordata (Willd.) R. Br. showed up in the European Food Safety Authority (EFSA) database. It is employed as a feed additive in intensive livestock farming in an effort to elevate daily food consumption and growth performance [19-24]. According to Mellor [25] and Le Floc'h and Seve [26], sanguinarine can regulate the serotonin synthesis by employing tryptophan and finally lead to improvement in feed intake [20]. However, more studies are required to investigate the effects of extract of Macleaya cordata (Willd.) R. Br. on pigs fed with tryptophan-deficient diet $[27,28]$.

Some investigations have revealed that dietary supplementation with isoquinoline alkaloids reduced the diarrhea and improved gut health, immune system, and digestive function in nonruminant mammals $[17,18,22,29]$. Therefore, the primary goal of this review was to discuss the impact of isoquinoline alkaloids, derived from extract of Macleaya cordata (Willd.) R. Br., on the growth and immune system in swine and poultry.

\section{The Impact of Isoquinoline Alkaloids, Derived from Extract of Macleaya cordata (Willd.) R. Br., on the Growth of Animals}

2.1. Swine. Phytobiotics can be defined as plant derived products added to feed in order to improve performance. It can be obtained through combining a large array of herbalbased products [30]. A number of researchers have claimed that some plants, as well as their extracts, are able to increase appetite and activate endogenous secretions of enzymes and hormones $[13,17,31]$. In the case of treatment of diseases, they have also been found to have the capacity to destroy microorganisms and parasitic worms in nonruminant animals. Moreover, they are able to retard the growth and reproduction of coccidian parasites [30].

Evidence is available from numerous studies to substantiate that adding phytochemical ingredients to the diet of pigs had beneficial outcomes, particularly in the treatment against growth retardation and disease. As antimicrobial agents, their efficacy is influenced by the concentration of additives and the $\mathrm{pH}$ in the animal's intestine [32]. Numerous researches revealed that phytochemical ingredients can reduce coliform bacteria in gastrointestinal tract (GIT) and decrease the diarrheal frequency or mortality rates among young pigs. Phytochemical additives also play an important role in deterring diarrhea and oedema in piglets during the weaning process [12].

Growth performance, as Kong et al. [13] and Jobgen et al. [33] stated, is a complicated progress involving the delicate interaction between metabolism and catabolism. But we may infer the potential physiological or biochemical effect of food additives on the animals through the investigation on the metabolites. For example, the metabolic properties of intracellular protein and the rate of fat deposits are valuable references for the determination of appropriate glucose and amino acid usage. It is no doubt that the metabolic processes are also modulated by hormones and other elements. Both antibiotics and extract of Macleaya cordata (Willd.) R. Br. can be used as growth promoters. When a comparative analysis was undertaken between them, the extract demonstrated similar effect as antibiotics on the intestinal health and growth performance $[17,29]$.

Using the extracts of Macleaya cordata (Willd.) R. Br. as feed additives at the concentration ranging from 15 to $50 \mathrm{mg} / \mathrm{kg}$, increased weight gain was found [17, 29]. This outcome has been attributed to the positive influence of internal and external factors on animal production, particularly due to their antimicrobial properties and their capacity to modify immune system and the reduction of inflammation [34]. A number of bacteria located in the mouth cavity of humans were identified to have antimicrobial qualities. Some of these bacteria were classified among the species frequently situated in the GIT of swine $[35,36]$. Feeding sanguinarine at minimal inhibitory concentration showed similar effect on bacteria. This may indicate that dietary supplements militate against the rapid multiplication of pathogen bacteria located in the GIT, which in turn impacts upon developmental progress.

From a scientific perspective, the primary contentious issue is about the effect of isoquinoline alkaloids from Macleaya cordata (Willd.) R. Br. on feed intake in farming animals. Some studies claimed that sanguinarine additives had no impact on feed consumption [28, 37]. Conversely, other researchers $[25,26]$ subscribed to the belief that sanguinarine could influence feed intake by regulating the pathway for the synthesis of serotonin by using tryptophan. One study showed that sanguinarine led to greater feed intake (increased by 7\%) and acquisition of nourishment, compared to those fed with antibiotics [17]. Beneficial effect on nitrogen balance and growth performance was also found when sanguinarine was added to the diet of swine [20].

No toxicity was found when swine and mice ingested the plant Macleaya cordata (Willd.) R. Br., let alone its alkaloid extract, because most of the possible contaminants had been removed [35, 38, 39]. Thus, adding the herb or/and its extract into animal feed would not expose the consumer to dangers. Furthermore, no negative impact on health was detected [35].

In addition, the introduction of isoquinoline alkaloids has decreased the prevalence of diarrhea [18]. Typically, diarrhea is associated with rapid multiplication of Escherichia coli and other pathogens in the intestine. The abnormal proliferation of bacteria results in the excretion of water and electrolytes through the semifluid feces and urine [13]. Isoquinoline alkaloids in the extract were found to suppress or destroy 
these microorganisms, as well as modulating vital functions, such as peristalsis and the $\mathrm{pH}$ of intestines [12].

Research conducted by Walker [8] and Newton et al. [9] confirmed that sanguinarine acts as an antimicrobial agent. They found that diet supplemented with sanguinarine had the potential to facilitate the establishment of beneficial bacteria in the GIT of swine, as well as the reinforcement of competitive exclusion principle by inhibiting the colonization of pathogenic bacteria. In addition, sanguinarine reduced the water loss in the epithelial cells of the intestines and/or enhanced the intestinal function in the absorption of water and nutrients [13]. The escalation of metabolic rates of biomolecules and the antioxidant capabilities in the small intestinal mucosa appeared to generate these effects [40].

One study demonstrated that the introduction of feed additives in the form of Macleaya cordata extract, containing isoquinoline alkaloids, increased the serum amino acids in swine [41]. And isoquinoline alkaloids can strengthen the capacity to assimilate and absorb ingested protein and AA. In addition, it is likely that this compound modulates the metabolism process in relation to the absorption of nutrients through signal transduction pathways. Nutrients augmentation in portal vein (and specifically AA) which derives from the small intestine may be adequate to stimulate tissue protein synthesis in animals, which has benefit impacts on the growth development $[40,42]$.

A correlation was found between feed additives and the enhanced movement of amino acids, leading to growth improvement. Greater volumes of essential amino acids, such as lysine, shield the intestine from pathogens and perform a crucial function in calcium absorption. They are also helpful in the preparation of muscle protein, hormones, enzymes, and antibodies [43, 44]. For example, arginine participates in various pathways, including the production of proteins, nitric oxide, polyamines, and creatine [45]. Methionine is another key intermediate in the biosynthesis of proteins and phospholipids. In addition, this amino acid, along with choline, contributes to transfer fat, thus decreasing the fat levels in liver. Methionine also has antioxidant property, and it comprises the element sulfur, which assists in neutralizing free radicals which emerge as a consequence of the diverse components of metabolism [40].

2.2. Poultry. Antibiotics as growth promoters have been withdrawn from the feedstuffs of poultry in most regions of the world. Therefore, an increasing demand for the exploration of other possible options is arising to sustain growth development. It is also important to ensure that beneficial microorganisms are predominant in the intestine to specifically prevent the proliferation of pathogenic bacteria. A number of plant additives have been extensively utilized to sustain or enhance the growth performance in poultry [46]. In addition, herb extracts may boost their immune system and decrease blood cholesterol levels [47].

Research has demonstrated that isoquinoline alkaloids prevent the spread of specific bacteria that generate gastrointestinal distress [48]. They also improve appetite and the growth performance [20]. In the case of broiler chickens and maturing turkeys, the recommended dose of Macleaya cordata in diet is 20 to $50 \mathrm{ppm}$ [49].

Variations have emerged in the studies conducted to measure the impact of isoquinoline alkaloids on broiler chickens. One study found that when chickens (Cobb $\times$ Cobb, male) ingested isoquinoline alkaloids at the dose of 25 and $50 \mathrm{ppm}$, the body mass and feed conversion rate increased [36]. Notwithstanding this, another research focusing on maturing Ross 308 chickens did not reach a similar conclusion. It found that isoquinoline alkaloids administration at $20 \mathrm{mg} / \mathrm{kg}$ failed to influence the growth development and the protein utilization in the poultry [50].

Nevertheless, the introduction of isoquinoline alkaloids into the diet has been claimed to have impact on gastrointestinal performance and the fermentation metabolic process in terminal GIT. It has also been confirmed that isoquinoline alkaloids influence the gastrointestinal movements [51]. Jankowski et al. [52] reported that adding Macleaya cordata compounds to the diet of broiler chickens could decrease inordinate fermentation in the caecum without disturbing the $\mathrm{pH}$ levels in this area, leading to the enhancement of growth performance.

\section{The Effects of Isoquinoline Alkaloids, Derived from Macleaya cordata Extract, on the Innate Immune Response}

3.1. Swine. Young pigs, weaned prior to the usual period (ranging from 15 to 28 days old), were subjected to situational tension and nutritional deficits, resulting in the rapid multiplication of intestinal disease-inducing bacteria (e.g., Escherichia coli). In addition to growth retardation, it led to higher morbidity and mortality rates $[13,53]$. This demonstrates that the innate immune system operating in young animals influences their performance levels, as well as their response to stimuli.

The innate immune system is an important subsystem of the overall immune system that comprises the cells and mechanisms that defend the host from infection by another organism. This implies that, within this immune system, cells identify and react to pathogen in a nonspecific manner. In contrast to the acquired immune system, it cannot endow immunity over a prolonged time period or defend its host. This innate immune system offers instantaneous protection from disease [54].

Throughout this phase, instantaneous defense is ensured by stimulating the inherent immune cells macrophages, as well as other cells such as the dendritic, polymorphonuclear, and epithelial. This occurs as a result of various toll-like receptors which identify crucial molecules on the outer layer of the bacteria [55]. Neutrophilic granulocytes consist of lysozyme, in primary as well as secondary granules. Its key role is to defend against pathogens and various foreign bodies surrounding the host [55]. This process is fully accomplished through phagocytosis and digesta. Therefore, the ingestion of isoquinoline alkaloids contained in extract of Macleaya cordata (Willd.) R. Br. was considered to be essential throughout crucial developmental phases, especially while the species is 
primarily dependent on intrinsic immunity [35]. The compound activates phagocytes, hence stimulating the organism's defense mechanisms [29].

Intestinal barrier systems are rigorously managed by a meticulously construed epithelial junctional complex, commonly known as the "the tight junction" [56]. It comprises a number of different proteins, which include a transmembrane protein called occludin [57], various derivatives of the claudin group, a junctional adhesion molecule [58], and several linker proteins, for example, $\mathrm{ZO}-1$. Three of the most crucial and beneficial proteins are occludin, ZO-1, and claudin-1, as they play an important role in the control of the tight junctions [59]. In connecting the C-terminal selections of $\beta$-actin and occludin [18], ZO-1 is a helpful linker protein in the tight junction.

Research has found that the ingestion of extract of Macleaya cordata (Willd.) R. Br. can increase the expression of ZO-1 and claudin-1. Thus, it is helpful in preventing allergenic and toxic matter entering the intestines and reducing risks [60]. This is indicates that the use of extract of Macleaya cordata (Willd.) R. Br. as a feed additive can promote intestinal mucosal growth and improve defense systems [18].

Recent research conducted by Kantas et al. [29] revealed that the introduction of alkaloids into the feedstuffs reduced the haptoglobin level in swine. This protein is found in blood plasma. It usually binds free hemoglobin and forms the hemoglobin-haptoglobin complex. Then the complex is withdrawn from circulation by the liver, whereupon it participated in a catabolic process in hepatic parenchymal cells. This study also demonstrated that dietary supplementation with alkaloids reduced the level of serum amyloid A (SAA). These proteins are a group of apolipoproteins produced in reaction to cytokines, which are stimulated by monocytes or macrophages. They are closely associated with inherent immunity. The long-established belief was that SAA performed a crucial function in relation to the biological mechanisms that lead to disease in amyloid A-type amyloidosis [61]. Hence, dietary supplementation with isoquinoline alkaloids extracted from Macleaya cordata (Willd.) R. Br. boosts the immune system and regulates metabolic process and finally promotes growth and development in swine.

3.2. Poultry. Antibiotics are usually replaced with probiotic, organic acids, and herbal extracts in poultry diet. Thus, it was necessary to clearly define the function of these compounds. Given these concerns, Yakhkeshi et al. [62] conducted a comparative analysis to determine the impact of herbal extracts, probiotics, organic acid, and antibiotics on the serum lipids, immune response, intestinal structures, and microbial population in broilers. No substantial variations were found in weight gain and feed conversion ratio when broilers were aged 1-14 and 14-28 days.

Furthermore, this study found that the alkaloids contained in these compounds substantially enhanced intestinal health and the absorption of nutrients. Unlike other interventions, the addition of sanguinarine to the diet resulted in a substantial rise in the heterophils to lymphocyte ratio $(\mathrm{H} / \mathrm{L})$. Evidence has shown that herbal extracts improve antibody titration against sheep red blood cells (SRBC). Studies have also demonstrated that herbal extracts trigger the immune system by boosting vitamin $\mathrm{C}$ levels. It has been recognized that isoquinoline alkaloids have the capacity to adjust or regulate immune functions [14]. In addition, this medicinal compound can activate phagocytosis, hence prompting defensive reactions by the host [63].

The introduction of isoquinoline alkaloids to the diet of broilers has been shown to considerably reduce the villus height of intestine and the depth of glandular layer [52]. But Vieira et al.[36] found no significant differences in villus height and crypt depth in broilers fed with and without sanguinarine. As far as we know, villus height and intestinal surface area are positively correlative to nutrients absorption and health in animals [64]. It was noted that cells situated in the villi (such as inflammatory cells or enterocytes) are also important when health problems exist. Typically, a greater volume of goblet and immunocyte are not directly correlated with nutrient absorption. But they were found to decrease absorption levels due to enhanced intestinal viscosity and the rate of passage of feeds.

Research undertaken by Pickler et al. [64] showed that the decrease of CD3 cells (this indicator relates to T lymphocytes cells) was detected in the duodenum, jejunum, and ileum of broilers fed with sanguinarine. More goblet cells were noted in the duodenum and ileum in control group compared with the group fed with sanguinarine. Sanguinarine was also found to alleviate the injury of mucosa, suggesting that it would be helpful to prevent enterobacterial infection.

This review has highlighted the idea that dietary supplementation with isoquinoline alkaloids, the extract of Macleaya cordata (Willd.) R. Br., is beneficial to swine and poultry. This compound increases feed consumption, body mass, and weight gain, as well as the concentration of serum amino acids. It boosts the innate immune system by regulating phagocytes, haptoglobin, and amyloid $\mathrm{A}$. In addition, it promotes effective gastrointestinal movements, as well as carrying out an important intestinal barrier function by action of ZO-1 protein and claudin-1.

\section{Competing Interests}

The authors declare that they have no competing interests.

\section{Authors' Contributions}

Hengjia Ni and Yordan Martínez contributed equally to this manuscript.

\section{Acknowledgments}

This study was in part supported by National Key Research and Development Program of China (2016YFD0500504), International Partnership Program of Chinese Academy of Sciences (161343KYSB20160008), the Science and Technology Department of Hunan Province (13JJ2034, 2013FJ3011, 2014NK3048, 2014NK4134, and 2014WK2032), National Natural Science Foundation of China (nos. 31330075, 
31110103909, 31572416, 31402092, 31501965, and 31372326), National Basic Research Program of China (2013CB127302, 2013CB127301), the Ministry of Agriculture 948 Program (2016-X47, 2015-Z64), and Chinese Academy of Sciences Visiting Professorship for Senior International Scientists Grant no. 2016VBB007.

\section{References}

[1] F. Zhang, B. Chen, S. Xiao, and S.-Z. Yao, "Optimization and comparison of different extraction techniques for sanguinarine and chelerythrine in fruits of Macleaya cordata (Willd) R. Br," Separation and Purification Technology, vol. 42, no. 3, pp. 283290, 2005.

[2] Z. Ming, L. Gui-Yin, Z. Jian-Guo et al., "Evaluation of molluscicidal activities of benzo[c]phenanthridine alkaloids from Macleaya cordata (Willd) R. Br. on snail hosts of Schistosoma japonicum," Journal of Medicinal Plants Research, vol. 5, no. 4, pp. 521-526, 2011.

[3] P. Kosina, J. Gregorova, J. Gruz et al., "Phytochemical and antimicrobial characterization of Macleaya cordata herb," Fitoterapia, vol. 81, no. 8, pp. 1006-1012, 2010.

[4] E. Vrublova, J. Vostalova, J. Ehrmann et al., "The phytogenic feed additive Sangrovit modulates dextran sulfate sodiuminduced colitis in rats," Veterinarni Medicina, vol. 55, no. 12, pp. 610-618, 2010.

[5] A. Šedo, K. Vlašicová, P. Barták et al., "Quaternary benzo[c]phenanthridine alkaloids as inhibitors of aminopeptidase $\mathrm{N}$ and dipeptidyl peptidase IV," Phytotherapy Research, vol. 16, no. 1, pp. 84-87, 2002.

[6] H. Chang and P. But, Pharmacology and Applications of Chinese Materia Medica, World Scientific, Singapore, 1987.

[7] V. Simanek, R. Vespalec, A. Sedo, J. Ulrichova, and J. Vicar, "Quaternary benzo[c]phenanthridine alkaloids-biological activities," in Chemical Probes in Biology, M. P. Schneider, Ed., vol. 129 of NATO Science Series II. Mathematics, Physics and Chemistry, pp. 245-254, Kluwer Academic, Dordrecht, The Netherlands, 2003.

[8] C. Walker, "Effects of sanguinarine and Sanguinaria extract on the microbiota associated with the oral cavity," Journal of the Canadian Dental Association, vol. 56, no. 7, pp. 13-30, 1990.

[9] S. M. Newton, C. Lau, S. S. Gurcha, G. S. Besra, and C. W. Wright, "The evaluation of forty-three plant species for in vitro antimycobacterial activities; isolation of active constituents from Psoralea corylifolia and Sanguinaria canadensis," Journal of Ethnopharmacology, vol. 79, no. 1, pp. 57-67, 2002.

[10] K. Wang, C. Luo, H. Liu, J. Xu, W. Sun, and L. Zhou, "Nematicidal activity of the alkaloids from Macleaya cordata against certain nematodes," African Journal of Agricultural Research, vol. 7, no. 44, pp. 5925-5929, 2012.

[11] M. M. Chaturvedi, A. Kumar, B. G. Darnay, G. B. N. Chainy, S. Agarwal, and B. B. Aggarwal, "Sanguinarine (pseudochelerythrine) is a potent inhibitor of NF- $\kappa \mathrm{B}$ activation, $\mathrm{I} \kappa \mathrm{B} \alpha$ phosphorylation, and degradation," Journal of Biological Chemistry, vol. 272, no. 48, pp. 30129-30134, 1997.

[12] L. L. Li, F. G. Yin, B. Zhang et al., "Dietary supplementation with Atractylodes Macrophala Koidz polysaccharides ameliorate metabolic status and improve immune function in earlyweaned pigs," Livestock Science, vol. 142, no. 1-3, pp. 33-41, 2011.

[13] X. F. Kong, G. Y. Wu, Y. P. Liao et al., "Dietary supplementation with Chinese herbal ultra-fine powder enhances cellular and humoral immunity in early-weaned piglets," Livestock Science, vol. 108, no. 1-3, pp. 94-98, 2007.

[14] W. Windisch, K. Schedle, C. Plitzner, and A. Kroismayr, "Use of phytogenic products as feed additives for swine and poultry," Journal of animal science, vol. 86, no. 14, pp. E140-E148, 2008.

[15] Y. Y. Ding, C. H. Zhang, X. L. He, L. Huang, and Z. J. Yin, "Growth performance responses and indicators of gastrointestinal health in early weaned pigs fed Chinese Herbal Medicine Additives-supplemented diets," Journal of Animal and Veterinary Advances, vol. 10, no. 12, pp. 1580-1587, 2011.

[16] Y. M. Aguilar, O. M. Yero, G. Liu et al., "Effect of dietary supplementation with Anacardium occidentale on growth performance and immune and visceral organ weights in replacement laying pullets," Journal of Food, Agriculture and Environment, vol. 11, no. 3-4, pp. 1352-1357, 2013.

[17] G. Liu, G. Y. M. Aguilar, W. Ren et al., "Dietary supplementation with sanguinarine enhances serum metabolites and antibodies in growing pigs," Journal of Animal Science, vol. 94, supplement 3, pp. 75-78, 2016.

[18] G. Liu, G. Guan, J. Fang et al., "Macleaya cordata extract decreased diarrhea score and enhanced intestinal barrier function in growing piglets," BioMed Research International, vol. 2016, Article ID 1069585, 7 pages, 2016.

[19] C. Franz, R. Bauer, R. Carle et al., "Assesment of plants/herbs, plant/herb extracts and their naturally or synthetically produced components as 'additives' for use in animal production," Tech. Rep. CFT/EFSA/FEEDAP/2005/01 2005, 2006.

[20] K. A. Tschirner, A. Susenbeth, and S. Wolffram, "Influence of Sangrovit ${ }^{\circledR}$ supplementation on nitrogen balance and feed intake in growing pigs," in Proceedings of the 9th Symposium Vitamins and Additives in the Nutrition of Man and Animal, 45 pages, Friedrich Schiller University, Jena, Germany, 2003.

[21] M. D. Rawling, D. L. Merrifield, and S. J. Davies, "Preliminary assessment of dietary supplementation of Sangrovit ${ }^{\circledR}$ on red tilapia (Oreochromis niloticus) growth performance and health," Aquaculture, vol. 294, no. 1-2, pp. 118-122, 2009.

[22] K.-W. Lee, J.-S. Kim, S.-T. Oh, C.-W. Kang, and B.-K. An, "Effects of dietary sanguinarine on growth performance, relative organ weight, cecal microflora, serum cholesterol level and meat quality in broiler chickens," Journal of Poultry Science, vol. 52, no. 1, pp. 15-22, 2015.

[23] J. A. Aguilar-Hernández, J. D. Urías-Estrada, M. A. López-Soto et al., "Evaluation of isoquinoline alkaloid supplementation levels on ruminal fermentation, characteristics of digestion, and microbial protein synthesis in steers fed a high-energy diet," Journal of Animal Science, vol. 94, no. 1, pp. 267-274, 2016.

[24] J. Dršata, J. Ulrichová, and D. Walterová, "Sanguinarine and chelerythrine as inhibitors of aromatic amino acid decarboxylase," Journal of Enzyme Inhibition, vol. 10, no. 4, pp. 231-237, 1996.

[25] S. Mellor, "Natural appetizers from plants," Feed Mix, vol. 9, no. 1, pp. 29-31, 2001.

[26] N. Le Floc'h and B. Seve, "Biological roles of tryptophan and its metabolism: potential implications for pig feeding," Livestock Science, vol. 112, no. 1-2, pp. 23-32, 2007.

[27] Y. Henry, B. Sève, Y. Colléaux, P. Ganier, C. Saligaut, and P. Jégo, "Interactive effects of dietary levels of tryptophan and protein on voluntary feed intake and growth performance in pigs, in relation to plasma free amino acids and hypothalamic serotonin," Journal of animal science, vol. 70, no. 6, pp. 18731887, 1992. 
[28] R. Blank, B. Müller-Siegwardt, and S. Wolffram, "Sanguinarine does not influence availability or metabolism of tryptophan in pigs," Livestock Science, vol. 134, no. 1-3, pp. 24-26, 2010.

[29] D. Kantas, V. G. Papatsiros, P. D. Tassis, L. V. Athanasiou, and E. D. Tzika, "The effect of a natural feed additive (Macleaya cordata), containing sanguinarine, on the performance and health status of weaning pigs," Animal Science Journal, vol. 86, no. 1, pp. 92-98, 2015.

[30] J. Vidanarachchi, L. L. Mikkelsen, I. Sims, P. A. Iji, and M. Choct, "Phytobiotics: alternatives to antibiotic growth promoters in monogastric animal feeds," Recent Advances in Animal Nutrition in Australia, vol. 15, pp. 131-144, 2005.

[31] J. Gong, F. Yin, Y. Hou, and Y. Yin, "Review: Chinese herbs as alternatives to antibiotics in feed for swine and poultry production: potential and challenges in application," Canadian Journal of Animal Science, vol. 94, no. 2, pp. 223-241, 2014.

[32] J. R. Pluske, D. J. Hampson, and I. H. Williams, "Factors influencing the structure and function of the small intestine in the weaned pig: a review," Livestock Production Science, vol. 51, no. 1-3, pp. 215-236, 1997.

[33] W. S. Jobgen, S. K. Fried, W. J. Fu, C. J. Meininger, and G. $\mathrm{Wu}$, "Regulatory role for the arginine-nitric oxide pathway in metabolism of energy substrates," Journal of Nutritional Biochemistry, vol. 17, no. 9, pp. 571-588, 2006.

[34] J. Lenfeld, M. Kroutil, E. Marsalek, J. Slavík, V. Preininger, and V. Simánek, "Antiinflammatory activity of quaternary benzophenanthridine alkaloids from Chelidonium majus," Planta Medica, vol. 43, no. 2, pp. 161-165, 1981.

[35] P. Kosina, D. Walterová, J. Ulrichová et al., "Sanguinarine and chelerythrine: assessment of safety on pigs in ninety days feeding experiment," Food and Chemical Toxicology, vol. 42, no. 1, pp. 85-91, 2004.

[36] S. L. Vieira, O. A. Oyarzabal, D. M. Freitas et al., "Performance of broilers fed diets supplemented with sanguinarine-like alkaloids and organic acids," Journal of Applied Poultry Research, vol. 17, no. 1, pp. 128-133, 2008.

[37] Y. Henry, B. Sève, Y. Colléaux, P. Ganier, C. Saligaut, and P. Jégo, "Interactive effects of dietary levels of tryptophan and protein on voluntary feed intake and growth performance in pigs, in relation to plasma free amino acids and hypothalamic serotonin," Journal of Animal Science, vol. 70, no. 6, pp. 18731887, 1992.

[38] J. Psotova, R. Vecera, A. Zdarilova et al., "Safety assessment of sanguiritrin, alkaloid fraction of Macleaya cordata, in rats," Veterinarni Medicina, vol. 51, no. 4, pp. 145-155, 2006.

[39] M. Stiborova, J. Vostalova, A. Zdarilova et al., "Macleaya cordata extract and Sangrovit genotoxicity. Assessment in vivo," Biomedical papers of the Medical Faculty of the University Palacký, Olomouc, Czechoslovakia, vol. 152, no. 1, pp. 35-39, 2008.

[40] G. Wu, "Intestinal mucosal amino acid catabolism," Journal of Nutrition, vol. 128, no. 8, p. 1249, 1998.

[41] G. Liu, Y. Martínez, W. Ren et al., "Effect of dietary supplementation with samgiomarome on growth performance, diarrhea score and serum concentrations of amino acids in weanling pigs," Amino acids, vol. 45, no. 3, p. 602, 2013.

[42] Y. H. Cai, Y. M. Aguilar, L. Yu et al., "Effects of dietary supplementation of Lactobacillus plantarum on growth performance and serum concentration of amino acids in weaned piglets ," Animal Nutrition and Feed Technology, vol. 14, no. 3, pp. 411420, 2014.
[43] P. J. Reeds, D. G. Burrin, B. Stoll et al., "Enteral glutamate is the preferential source for mucosal glutathione synthesis in fed piglets," American Journal of Physiology-Endocrinology and Metabolism, vol. 273, no. 2, pp. 408-415, 1997.

[44] J. Wang, D. Li, L. J. Dangott, and G. Wu, "Proteomics and its role in nutrition research," Journal of Nutrition, vol. 136, no. 7, pp. 1759-1762, 2006.

[45] G. Wu and S. M. Morris Jr., "Arginine metabolism: nitric oxide and beyond," Biochemical Journal, vol. 336, no. 1, pp. 1-17, 1998.

[46] J. Gardzielewska, K. Pudyszak, T. Majewska, M. Jakubowska, and J. Pomianowski, "Effect of plant-supplemented feeding on fresh and frozen storage quality of broiler chicken meat," Animal Husbandry Series of Electronic Journal Polish. Agriculture University, vol. 6, no. 2, pp. 322-332, 2003.

[47] R. Mathivanan and K. Kalaiarasi, "Panchagavya and Andrographis paniculata as alternatives to antibiotic growth promoters on haematological, serum biochemical parameters and immune status of broilers," The Journal of Poultry Science, vol. 44, no. 2, pp. 198-204, 2007.

[48] G. B. Mahady, S. L. Pendland, A. Stoia, and L. R. Chadwick, "In vitro susceptibility of Helicobacter pylori to isoquinoline alkaloids from Sanguinaria canadensis and Hydrastis canadensis," Phytotherapy Research, vol. 17, no. 3, pp. 217-221, 2003.

[49] Z. Zdunczyk, R. Gruzauskas, J. Juskiewicz et al., "Growth performance, gastrointestinal tract responses, and meat characteristics of broiler chickens fed a diet containing the natural alkaloid sanguinarine from Macleaya cordata," The Journal of Applied Poultry Research, vol. 19, no. 4, pp. 393-400, 2010.

[50] K. Kozlowski, A. Lecewicz, H. Jeroch, Z. Zdunczyk, J. Jankowski, and K. Kozlowski, "Effect of a phytogenic feed additive from Macleaya cordata on performance and carcass parameters of broilers," Archiv fur Geflugelkunde, vol. 72, no. 3, pp. 140-142, 2008.

[51] C.-L. Wu, C.-R. Hung, F.-Y. Chang, L.-C. Lin, K.-Y. F. Pau, and P. S. Wang, "Effects of evodiamine on gastrointestinal motility in male rats," European Journal of Pharmacology, vol. 457, no. 2-3, pp. 169-176, 2002.

[52] J. Jankowski, Z. Zduńczyk, J. Juśkiewicz, K. Kozłowski, A. Lecewicz, and H. Jeroch, "Gastrointestinal tract and metabolic response of broilers to diets with the Macleaya cordata alkaloid extract," Archiv fur Geflugelkunde, vol. 73, no. 2, pp. 95-101, 2009.

[53] P. Liu, X. S. Piao, S. W. Kim et al., "Effects of chito-oligosaccharide supplementation on the growth performance, nutrient digestibility, intestinal morphology, and fecal shedding of Escherichia coli and Lactobacillus in weaning pigs," Journal of Animal Science, vol. 86, no. 10, pp. 2609-2618, 2008.

[54] M. F. Tosi, "Innate immune responses to infection," Journal of Allergy and Clinical Immunology, vol. 116, no. 2, pp. 241-249, 2005.

[55] P. Bourlioux, B. Koletzko, F. Guarner, and V. Braesco, "The intestine and its microflora are partners for the protection of the host: report on the Danone Symposium 'The Intelligent Intestine' held in Paris, June 14, 2002," American Journal of Clinical Nutrition, vol. 78, no. 4, pp. 675-683, 2003.

[56] T. Kucharzik, S. V. Walsh, J. Chen, C. A. Parkos, and A. Nusrat, "Neutrophil transmigration in inflammatory bowel disease is associated with differential expression of epithelial intercellular junction proteins," The American Journal of Pathology, vol. 159, no. 6, pp. 2001-2009, 2001.

[57] V. Wong and B. M. Gumbiner, "Synthetic peptide corresponding to the extracellular domain of occludin perturbs the tight 
junction permeability barrier," Journal of Cell Biology, vol. 136, no. 2, pp. 399-409, 1997.

[58] C.-J. Wu, P. Mannan, M. Lu, and M. C. Udey, "Epithelial cell adhesion molecule (EpCAM) regulates claudin dynamics and tight junctions," Journal of Biological Chemistry, vol. 288, no. 17, pp. 12253-12268, 2013.

[59] A. S. Fanning, B. J. Jameson, L. A. Jesaitis, and J. M. Anderson, "The tight junction protein ZO-1 establishes a link between the transmembrane protein occludin and the actin cytoskeleton," The Journal of Biological Chemistry, vol. 273, no. 45, pp. 2974529753, 1998.

[60] L. Montagne, G. Boundry, C. Favier, I. Le Huerou-Luron, J.-P. Lallès, and B. Sève, "Main intestinal markers associated with the changes in gut architecture and function in piglets after weaning," British Journal of Nutrition, vol. 97, no. 1, pp. 45-57, 2007.

[61] K. K. Eklund, K. Niemi, and P. T. Kovanen, "Immune functions of serum amyloid A," Critical Reviews in Immunology, vol. 32, no. 4, pp. 335-348, 2012.

[62] S. Yakhkeshi, S. Rahimi, and K. Gharib Naseri, "The effects of comparison of herbal extracts, antibiotic, probiotic and organic acid on serum lipids, immune response, GIT microbial population, intestinal morphology and performance of broilers," Journal of Medicinal Plants, vol. 10, no. 37, pp. 80-95, 2011.

[63] D. Gudev, S. Popova- Ralcheva, P. Moneva, M. Bonovska, G. Valchev, and A. Valcheva, "Effect of supplemental Sangrovit on some biochemical indices and leukocytes phagocytic activity in growing pigs," Archiva Zootechnica, vol. 7, pp. 123-134, 2004.

[64] L. Pickler, B. C. B. Beirão, R. M. Hayashi et al., "Effect of sanguinarine in drinking water on Salmonella control and the expression of immune cells in peripheral blood and intestinal mucosa of broilers," The Journal of Applied Poultry Research, vol. 22, no. 3, pp. 430-438, 2013. 


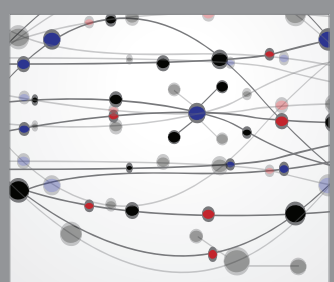

The Scientific World Journal
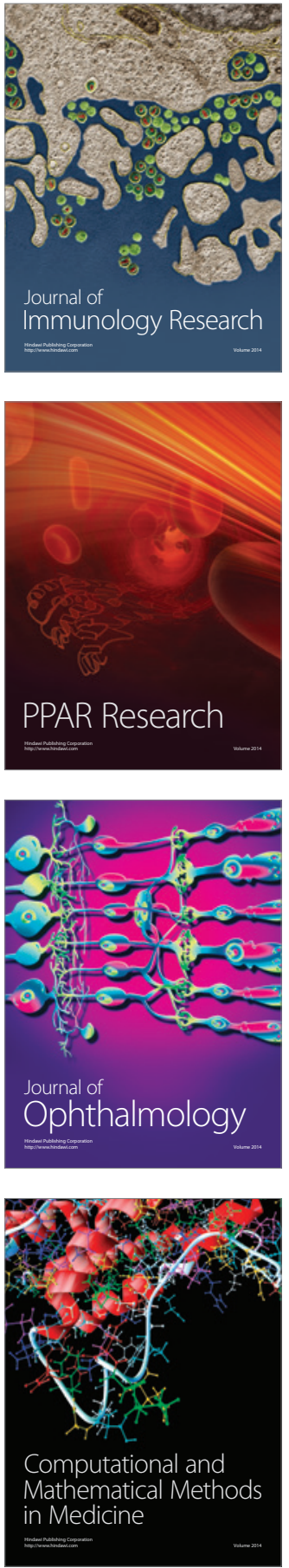

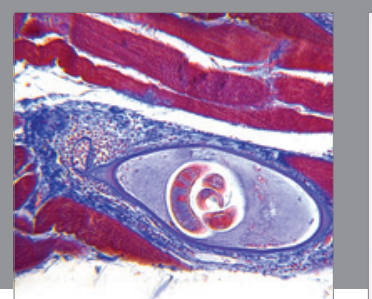

Gastroenterology Research and Practice

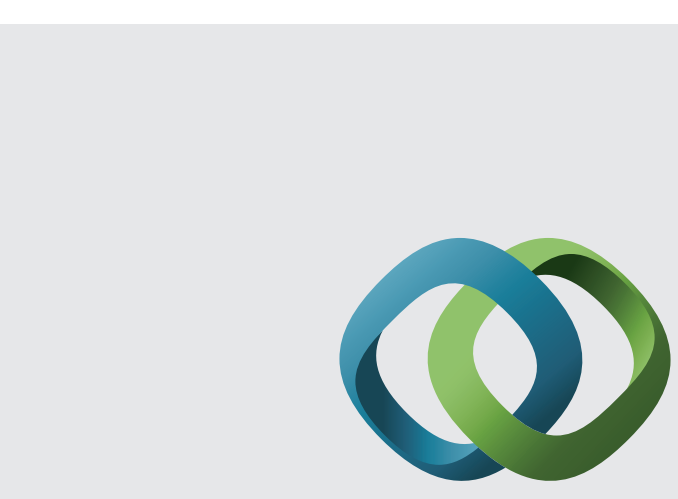

\section{Hindawi}

Submit your manuscripts at

http://www.hindawi.com
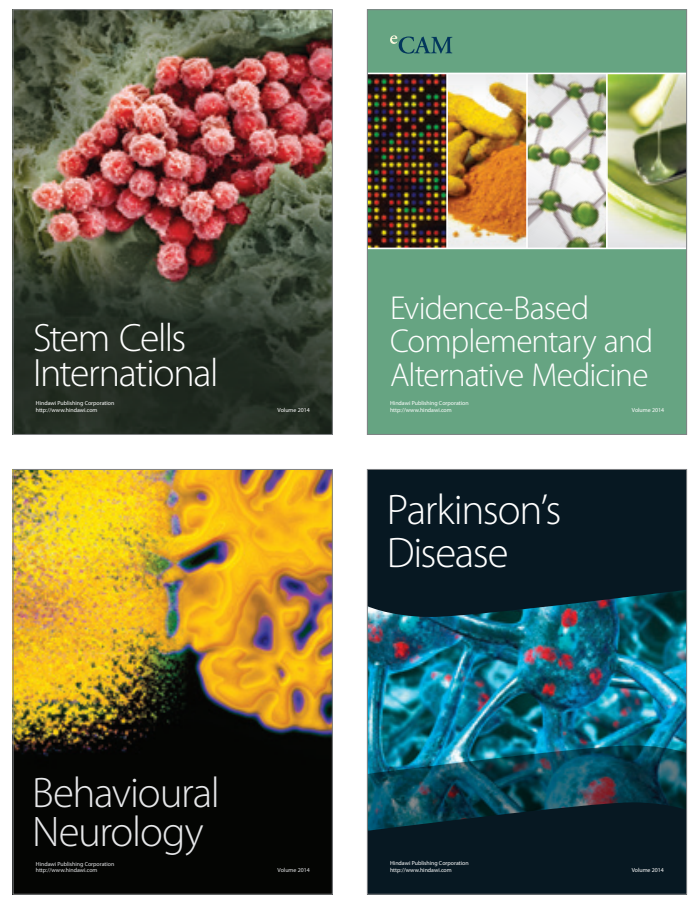
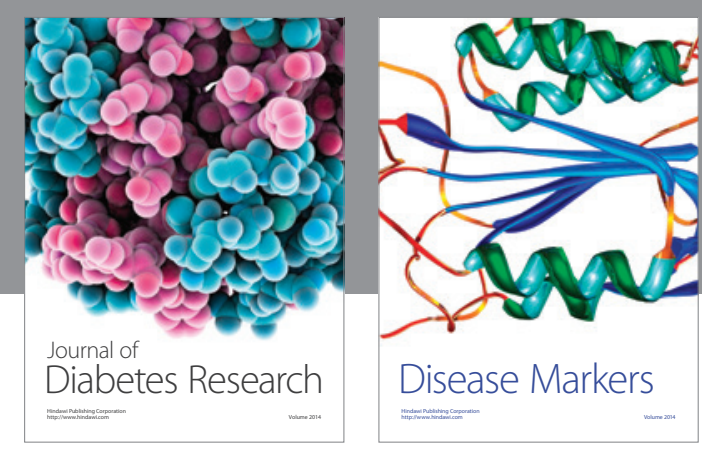

Disease Markers
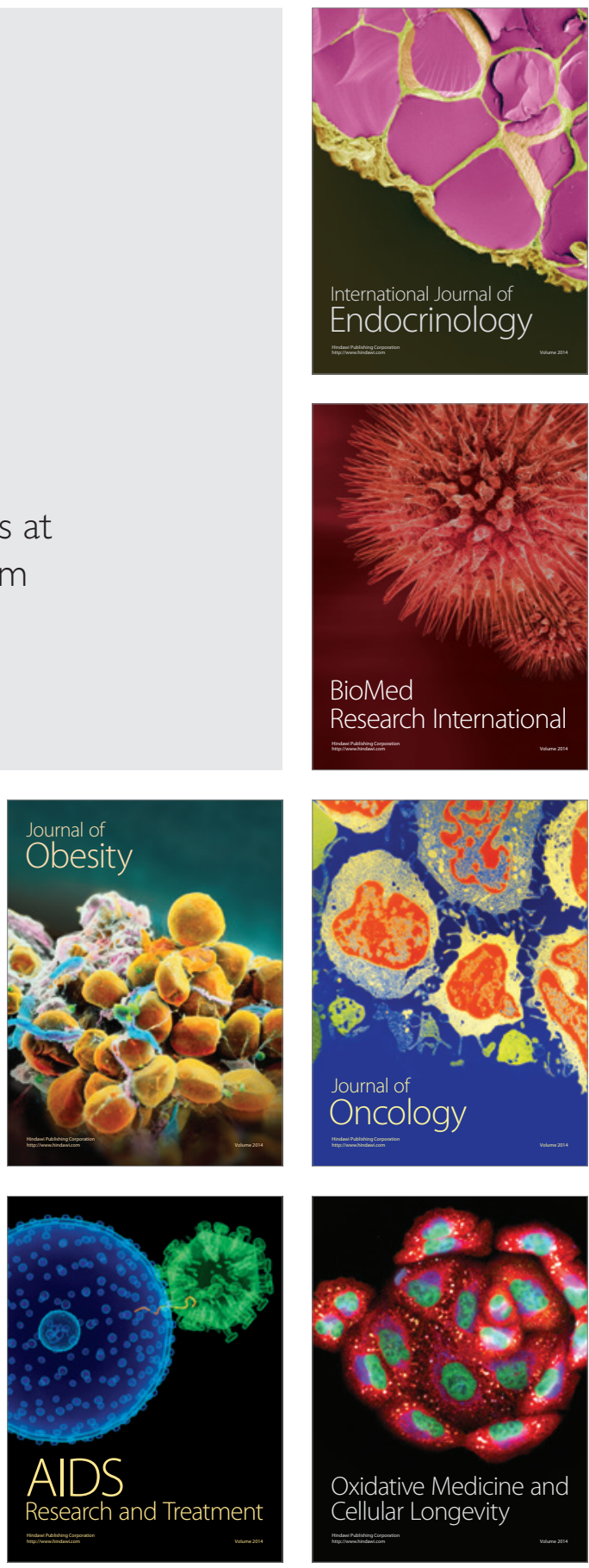